\title{
Bur open Error rates in a clinical data repository: lessons from the transition to electronic data transfer-a descriptive study
}

\author{
Matthew K H Hong, ${ }^{1}$ Henry $\mathrm{H}$ I Yao, ${ }^{1}$ John S Pedersen, ${ }^{2}$ Justin S Peters, ${ }^{1}$ \\ Anthony J Costello, ${ }^{1}$ Declan G Murphy, ${ }^{1,3}$ Christopher M Hovens, ${ }^{1}$ Niall M Corcoran ${ }^{1}$
}

To cite: Hong MKH, Yao HHI, Pedersen JS, et al. Error rates in a clinical data repository: lessons from the transition to electronic data transfer-a descriptive study. BMJ Open 2013;3:e002406.

doi:10.1136/bmjopen-2012002406

- Prepublication history for this paper are available online. To view these files please visit the journal online (http://dx.doi.org/10.1136/ bmjopen-2012-002406).

Received 26 November 2012 Accepted 9 April 2013

This final article is available for use under the terms of the Creative Commons Attribution Non-Commercial 2.0 Licence; see

http://bmjopen.bmj.com

\footnotetext{
${ }^{1}$ Division of Urology, Department of Surgery, University of Melbourne, Royal Melbourne Hospital and the Australian Prostate Cancer Research Centre Epworth, Melbourne, Victoria, Australia

${ }^{2}$ TissuPath Specialist Pathology, Mount Waverley and Monash University Faculty of Medicine, Melbourne, Victoria, Australia ${ }^{3}$ Division of Cancer Surgery, Peter MacCallum Cancer Centre, East Melbourne, Victoria, Australia
}

Correspondence to Dr Matthew Hong; m.k.hong@ausdoctors.net

\section{ABSTRACT}

Objective: Data errors are a well-documented part of clinical datasets as is their potential to confound downstream analysis. In this study, we explore the reliability of manually transcribed data across different pathology fields in a prostate cancer database and also measure error rates attributable to the source data.

Design: Descriptive study.

Setting: Specialist urology service at a single centre in metropolitan Victoria in Australia.

Participants: Between 2004 and 2011, 1471 patients underwent radical prostatectomy at our institution. In a large proportion of these cases, clinicopathological variables were recorded by manual data-entry. In 2011 , we obtained electronic versions of the same printed pathology reports for our cohort. The data were electronically imported in parallel to any existing manual entry record enabling direct comparison between them.

Outcome measures: Error rates of manually entered data compared with electronically imported data across clinicopathological fields.

Results: 421 patients had at least 10 comparable pathology fields between the electronic import and manual records and were selected for study. 320 patients had concordant data between manually entered and electronically populated fields in a median of 12 pathology fields (range 10-13), indicating an outright accuracy in manually entered pathology data in $76 \%$ of patients. Across all fields, the error rate was $2.8 \%$, while individual field error ranges from $0.5 \%$ to $6.4 \%$. Fields in text formats were significantly more error-prone than those with direct measurements or involving numerical figures $(p<0.001) .971$ cases were available for review of error within the source data, with figures of $0.1-0.9 \%$.

Conclusions: While the overall rate of error was low in manually entered data, individual pathology fields were variably prone to error. High-quality pathology data can be obtained for both prospective and retrospective parts of our data repository and the electronic checking of source pathology data for error is feasible.

\section{BACKGROUND AND SIGNIFICANCE}

The majority of clinical research publications are based on the analysis of prospectively or

\section{ARTICLE SUMMARY}

\section{Article focus}

- Although the use of structured electronic databases is widespread, a substantial amount of clinical data used in research predates this.

- There is a paucity of literature on error rates in such clinical datasets used in research.

- We explored the reliability of manually transcribed data across different pathology fields in a prostate cancer database and also measured error rates attributable to the source data.

Key messages

- While the overall rate of error for manually entered data can be low, individual fields may be variably prone to error, especially those involving descriptive text or requiring an element of interpretation.

- Computerised systems can be used to check clinical source data for error.

- The use of electronic data feeds retrospectively can replace manually collected data fields in some cases to improve overall accuracy.

Strengths and limitations of this study

- Our study design provides a realistic representation of a small-to-moderate-sized oncology database used for research purposes.

- We checked the integrity of one aspect of our source data.

- Our study was limited by its use of a single spreadsheet from a single series of patients.

- As we only examined the pathology fields covered by electronic import, the findings were not representative of the entire dataset.

retrospectively constructed, clinical databases. In addition, patient-centered databases are increasingly important in translational research efforts, as appropriately annotated tissue banks are the foundation for global multi-institutional collaborative efforts in genetic and epigenetic screening of various diseases. ${ }^{1}$ Yet, despite the stringent quality controls placed on the vast amounts of 
research data derived from these studies and the acute awareness of the need to control data quality, ${ }^{2}{ }^{3}$ the inherent accuracy of original clinical datasets is one area that receives relatively little attention.

Data errors are common in clinical datasets, ${ }^{4-6}$ with some cancer databases recording error rates as high as almost $27 \%$ in some fields. ${ }^{7}$ Such errors have the potential to adversely affect data analysis and interpretation, and can lead to erroneous conclusions. ${ }^{8}$ Methods to first identify and then correct errors in these datasets would be immensely valuable in the setting of the large-scale genomics projects being performed.

Two types of errors are described in the literature: one of omission and one of erroneous value. Although it is sometimes argued that missing values carry greater impact due to their greater prevalence, ${ }^{9}$ which may be up to $55 \%$ in cancer surgery databases, ${ }^{10}$ these errors are more easily detected with judicious computer queries and corrected with retrospective data collection. On the contrary, once erroneous values permeate a dataset, their effects can cascade in unpredictable ways. Errors in high impact fields have been shown to adversely affect the interpretation of statistical analyses, even if the errors are at low prevalence. ${ }^{11}$ While it is well known that structured data entry improves the accuracy of manual documentation, ${ }^{12}$ much of the clinical data of high value to researchers predates any effective informatics solutions aimed at data quality that might exist today. Instead, manual retrospective transcription of data from clinical records into relatively unstructured spreadsheets constitutes the data entry method for many clinical audits that subsequently serve research purposes. These datasets may have even transitioned to more carefully constructed data entry interfaces, as might occur in conditions such as prostate cancer where long follow-up times of over 10 years are necessary for study of oncological outcomes. ${ }^{13}$ In such cases, the provenance of the data collected with earlier means may not be accounted for with subsequent analysis.

Studies involving large cancer datasets rarely report error rates or their management, and it is difficult to assess the impact that these may have on the outcomes reported. ${ }^{14}$ Given the considerable effort that generally underlies the collection of data for a large clinical database, it is unsurprising that surplus resources are usually unavailable to place towards the check of data accuracy. Although larger numbers in databases may be used to counter the problem of errors, the combination of datasets, particularly with different fields would serve only to magnify error rates.

Knowledge of errors in manually collected data could give insight into how these may be accounted for in subsequent analysis. In cancer databases, pathology data are of particular importance as they are relied upon to build cohorts of clinical relevance in research. There are often multiple fields that give equivalent indications of underlying biology and any one could be analysed to similar effect. For example, either the percentage involvement of tumour or its measured size may serve as parameters of cancer burden. It is unlikely that different types of data would have equivalent vulnerability to error, and knowledge of the fields or types of fields that might be more error-prone with manual data entry could help researchers judiciously select fields for analysis based on greater accuracy. It might also aid informaticians to focus on error prevention in fields that carry particular importance in clinical and research settings. In addition, it is important to measure the baseline level of error inherent within the pathology reports themselves, the data source, as no degree of accuracy in manual transcription or even automated processes can result in a lower error rate without amendment of the original report.

In this article, we explored the reliability of manually transcribed pathology data across different fields in a large contemporary prostate cancer database. Initially housed as a Microsoft Excel spreadsheet, the database has evolved to become server based with a web-based interface. We have established automated electronic datafeeds from our pathology service provider to reduce the manual human data entry component in the pathology data, and we have used these to prospectively and retrospectively populate data fields. We compared the overlapping data from the electronic feeds and previously manually entered data, whereby we could gauge the accuracy of pathology details in a subset of our patients. In this way, we could determine the error involved in manual data entry in different fields across patients with relative ease. In addition, we explored the error that might be attributable to the source data.

\section{PATIENT AND METHODS}

\section{Database systems and data linking}

Between 2004 and 2011, 1471 patients underwent radical prostatectomy across our institutions. Fifty seven fields per patient, including demographics, preoperative prostate-specific antigen, pathological Gleason score and stage and other pathological data relating to the prostatectomy specimens were manually entered in a nonrelational database (Microsoft Excel spreadsheet) for the first 853 of these cases (2004-2008). Although most data collection was primarily prospectively performed, pathology data were obtained retrospectively once printed specimen pathology reports became available, or missing data were found on later review. For every patient, printed pathology reports were consulted and data were manually transcribed into the spreadsheet. Each of the reports were issued by a single pathology group and consisted of one to two pages of prose. Since 2006, the reports have been accompanied by a separate page with a 'synoptic' report. This synoptic report contained the pathology data in a structured format with fields of interest listed on a single page enabling greater ease of interpretation over the traditional reports in prose. Manual data entry was performed by four surgical residents with 
knowledge of prostate cancer pathology and versed in the relevant terminology. In 2010, our institution moved all data to Caisis V.5.0, a web-based relational database system developed at the Memorial Sloan Kettering Cancer Centre in New York, and ceased manual recording of pathology data from hardcopy reports.

We subsequently established a data link between our database and the pathology group whereby electronically encrypted reports were provided in HL7 standard V.2.31 format, a health industry information technology standard. The reports were retrieved using client/server software through a TCP/IP link. Custom software was developed in Visual Basic (Microsoft Visual Studio 2010) that enabled us to parse text or values of interest from the synoptic reports and automatically populate associated fields in our database system (figure 1). All new pathology data henceforth were imported in this way after testing of the new software for accuracy was performed. Digital import of data was conservative with the software being written in such a way that data were not transferred in cases of ambiguity. Given that the original synoptic reports dating back to 2006 were digitally stored by our pathology group, we were able to import pathology data from this time period with our software and use it in preference to any data arising from manual data entry for patients from 2006 to 2011.

\section{Analysis of error in manual data entry}

The digitally imported data were placed parallel to any existing manual entry records; following institutional review board approval, we were able to directly compare between digitally imported data and manual entry data for 752 patients where records from the two data entry methods coexisted. We assumed that any mismatch in the fields within the manual entry and digital import was due to human error in the data entry, as data were copied from printed versions of these same reports in the first instance. The importing software had been extensively tested and errors would be systematic within each field rather than transcriptional in nature. We excluded from analysis specimen pathology fields with fewer than 200 comparable entries in order to detect at least a $0.5 \%$ error rate. Four hundred and twenty-one patients had at least 10 completed pathology fields in both manual entry and electronic import records and were thus selected for study. This would allow us to detect the error rate across a patient's fields and also minimise individual patient factors in explaining error rates in different fields.

Within each pathology field, we linked the records based on unique identifiers and electronically compared them using custom-prepared software (Visual Basic, Microsoft Visual Studio 2010). We identified and counted any mismatches and then compared across all fields for each patient to determine the number of patients affected by one or more errors across the cohort (figure 2).

\section{Analysis of error within pathology reports}

In order to gain insight into the error inherent within the original pathology reports from which we sourced data, we measured the concordance between pathological stage and one of the descriptors that led to stage determination, namely the extraprostatic extension variable. An error was detected in cases of incongruity where extraprostatic extension was present but the

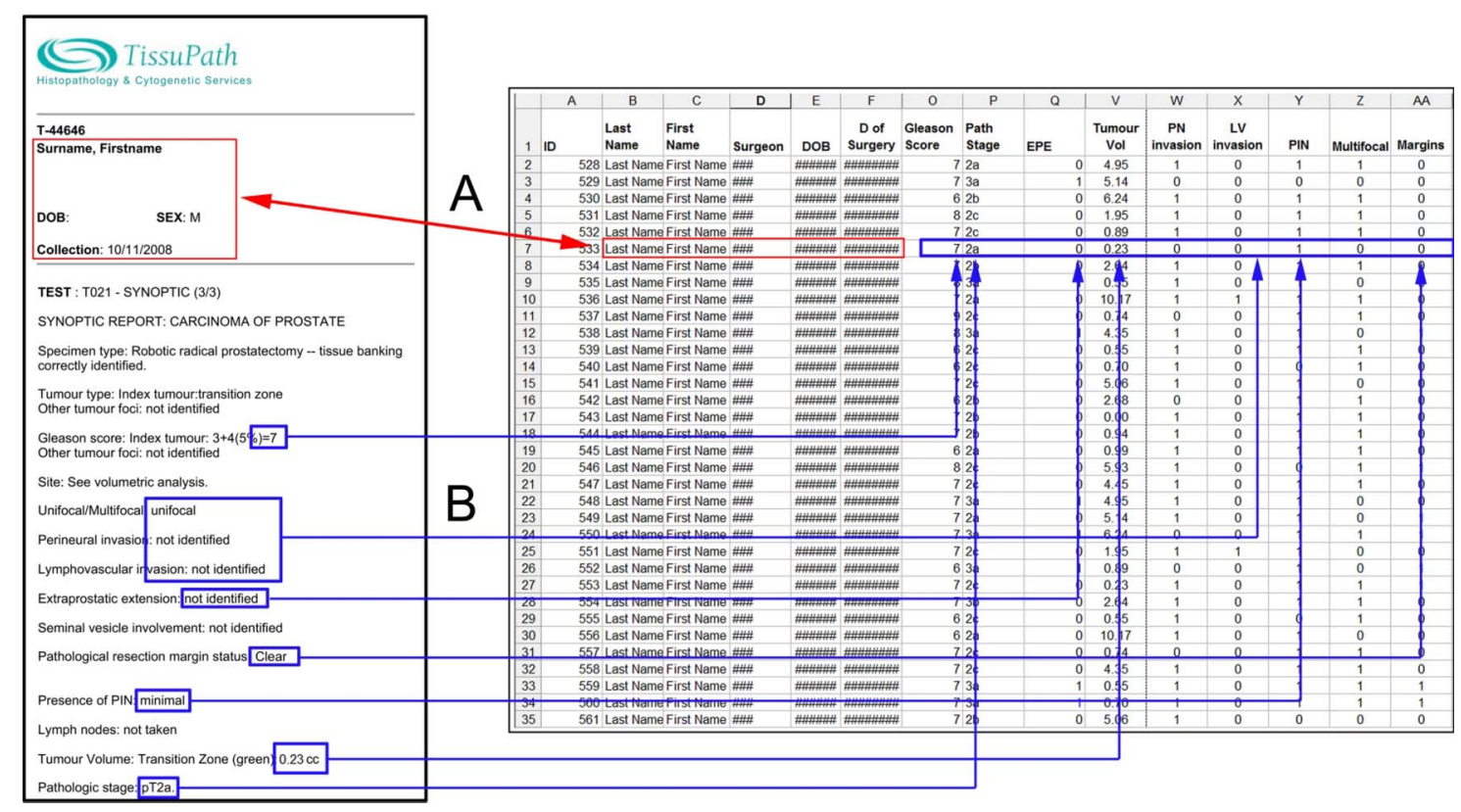

Figure 1 Schematic representation of the digital import of pathology data. Structured 'synoptic' reports facilitated digital recognition of relevant pathology fields. (A) Demographics data were used to link reports to individual patients in the database and $(B)$ individual data were then extracted from the report and directed to populate relevant fields in the main database. 
Figure 2 Schematic representation of the comparison of a dataset imported digitally and in parallel to a manually entered dataset. (A) Records were linked using unique patient identifiers and (B) pathology fields were individually compared.

Concordant data were flagged for merging in order to eliminate duplicate data. (C) Mismatches were used to identify errors in the manual entry dataset. (D) We compared across all pathology fields for individual patients.

\begin{tabular}{|c|c|c|c|c|c|c|c|c|c|}
\hline & A & 0 & $\mathrm{P}$ & Q & & A & 0 & $\mathrm{P}$ & Q \\
\hline 1 & ID & $\begin{array}{l}\text { Gleason } \\
\text { Score }\end{array}$ & $\begin{array}{l}\text { Path } \\
\text { Stage }\end{array}$ & EPE & 1 & ID & $\begin{array}{l}\text { Gleason } \\
\text { Score }\end{array}$ & $\begin{array}{l}\text { Path } \\
\text { Stage }\end{array}$ & EPE \\
\hline 2 & 528 & $1<$ & $2 a$ & -0 & 2 & 528 & & $2 a$ & 0 \\
\hline 3 & 529 & 7 & $3 a$ & 1 & 3 & 529 & & $3 a$ & 1 \\
\hline 4 & 530 & 6 & $2 b$ & 0 & 4 & 530 & & $2 b$ & 0 \\
\hline 5 & 531 & 8 & $2 c$ & 0 & 5 & 531 & & $\sqrt[3]{2 c}$ & c \\
\hline 6 & 532 & 7 & $2 c$ & 0 & 6 & 532 & & $2 c$ & 0 \\
\hline 7 & 533 & 7 & 2c & 0 & 7 & 533 & & $2 c$ & 0 \\
\hline 8 & 534 & 7 & $2 b$ & 0 & 8 & 534 & 7 & $2 \mathrm{~b}$ & c \\
\hline 9 & 535 & 8 & $3 a$ & 1 & 9 & 535 & & $3 a$ & 1 \\
\hline 10 & 536 & 7 & $2 a$ & 0 & 10 & 536 & & $2 a$ & 0 \\
\hline 11 & 537 & 9 & 2c & 0 & 11 & 537 & & $2 \mathrm{c}$ & 0 \\
\hline 12 & 538 & 8 & $3 a$ & 1 & 12 & 538 & & $3 a$ & 1 \\
\hline 13 & 539 & 6 & $2 c$ & 0 & 13 & 539 & & $2 c$ & 0 \\
\hline 14 & 540 & 6 & $2 c$ & 0 & 14 & 540 & & $2 c$ & c \\
\hline 15 & 541 & 7 & $2 c$ & 0 & 15 & 541 & & $2 c$ & c \\
\hline 16 & 542 & 6 & $2 b$ & 0 & 16 & 542 & & $2 b$ & 0 \\
\hline 17 & 543 & 7 & $2 b$ & 0 & 17 & 543 & & $2 \mathrm{~b}$ & c \\
\hline 18 & 544 & 7 & $2 b$ & 0 & 18 & 544 & & $2 \mathrm{~b}$ & c \\
\hline 19 & 545 & 6 & $2 a$ & 0 & 19 & 545 & & $2 a$ & c \\
\hline 20 & 546 & 8 & $2 c$ & 0 & 20 & 546 & & $3 \mathrm{c}$ & c \\
\hline 21 & 547 & 7 & $2 \mathrm{c}$ & $\theta$ & 24 & 547 & & 2 & c \\
\hline 22 & 548 & 7 & $3 a$ & 1 & 22 & 548 & & $3 a$ & 1 \\
\hline 23 & 549 & 7 & $2 a$ & 0 & 23 & 549 & & $2 a$ & c \\
\hline 24 & 550 & 7 & $3 a$ & 1 & 24 & 550 & & $3 a$ & 1 \\
\hline 25 & 551 & 7 & $2 c$ & 0 & 25 & 551 & & $2 \mathrm{c}$ & 0 \\
\hline 26 & 552 & 6 & $3 a$ & 1 & 26 & 552 & & $3 a$ & 1 \\
\hline 27 & 553 & 7 & $2 c$ & 0 & 27 & 553 & & $2 c$ & c \\
\hline 28 & 554 & 7 & $3 b$ & 0 & 28 & 554 & & $3 b$ & 0 \\
\hline 29 & 555 & 6 & $2 c$ & 0 & 29 & 555 & & $2 c$ & 0 \\
\hline 30 & 556 & 6 & $2 a$ & 0 & 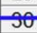 & -550 & & $2 a$ & 0 \\
\hline 31 & 557 & 7 & $2 c$ & 0 & 31 & 557 & & $2 \mathrm{c}$ & 0 \\
\hline 32 & 558 & 7 & $2 c$ & 0 & 32 & 558 & & $2 c$ & c \\
\hline 33 & 559 & 7 & $3 a$ & 1 & 33 & 559 & & $3 a$ & 1 \\
\hline 34 & 560 & 7 & $3 a$ & 1 & 34 & 560 & & $3 a$ & 1 \\
\hline 35 & 561 & 7 & $2 b$ & 0 & 35 & 561 & & $2 \mathrm{~b}$ & 0 \\
\hline
\end{tabular}

staging was T2, or extraprostatic extension was absent but the staging was T3. We identified and excluded the small number of cases of stage T3b disease where seminal vesicle invasion was apparent but extraprostatic extension not definite, as this would not be considered an error. We examined only the electronically imported data read directly from the synoptic reports, so that the effect of manual data entry was excluded. We identified a total of 971 cases where both pathological stage and extraprostatic extension fields had both been successfully imported from the synoptic pathology reports (table 1). Once again, using Visual Basic, we generated

\begin{tabular}{lr} 
Table 1 Digitally imported cases containing pathological \\
stage and extraprostatic extension variables where \\
comparison enabled analysis of error within the source \\
pathology reports & Number \\
\hline Pathological stage & 131 \\
\hline T2a & 4 \\
T2b & 543 \\
T2c & 225 \\
T3a & 68 \\
T3b & 0 \\
T4 & \\
Extraprostatic extension & 670 \\
Absent & 302 \\
Present & 971 \\
Total cases for comparison
\end{tabular}

a report indicating cases where there was a mismatch between pathological stage and extraprostatic extension status. The original cases in which these mismatches occurred were all reviewed by a pathologist to confirm the presence of error in the source material.

\section{Statistical analysis}

Percentage error rates were calculated by dividing the absolute number of errors by the total number of data points examined overall and in each field. Binomial distribution was used to calculate $95 \%$ CIs for these rates, and Fisher's exact test applied to $2 \times 2$ contingency tables where necessary (PASW Statistics V.18.0; IBM, Chicago, Illinois, USA, 2010). All statistical tests were two-tailed and significance was assumed at $\alpha<0.05$.

\section{RESULTS}

Of the 421 patients selected for this part of the study, 320 had completely concordant data between manual entry and electronic import methods in a median of 12 pathology fields (range 10-13), indicating an outright accuracy in $76 \%$ of all patients. Seventy-one patients $(16.8 \%)$ had errors in one field only, while $18(4.3 \%)$ had two or more incorrect fields and $12(2.9 \%)$ had $3-5$ errors.

Analysis of error rate in each individual pathology field yielded rates of error ranging from $0.5 \%$ to $6.4 \%$. Across all fields, the error rate was $2.8 \%$ (table 2). Assuming that errors in different fields occurred independently of one another, the fraction of records where at least one error occurred would be given by 1 
Table 2 Analysis of error rate in individual pathology fields in a radical prostatectomy dataset

\begin{tabular}{|c|c|c|c|c|c|c|}
\hline Pathology field & Variable type & Data format & Total data points & Error & Error rate (\%) & $(95 \% \mathrm{Cl})$ \\
\hline Gleason 1 & Categorical & Numeric & 415 & 2 & 0.5 & (0.06 to 1.7$)$ \\
\hline Gleason 2 & Categorical & Numeric & 415 & 3 & 0.7 & (0.15 to 2.1$)$ \\
\hline Gleason score & Categorical & Numeric & 415 & 1 & 0.2 & (0.01 to 1.3$)$ \\
\hline Extraprostatic extension* & Binary & Text & 421 & 21 & 5.0 & (3.1 to 7.5 ) \\
\hline Stage & Categorical & Alphanumeric & 421 & 13 & 3.1 & (1.7 to 5.2$)$ \\
\hline Focality & Binary & Text & 421 & 9 & 2.1 & (1.0 to 4.0$)$ \\
\hline Perineural invasion* & Categorical & Text & 421 & 27 & 6.4 & (4.3 to 9.2 ) \\
\hline Lymphovascular invasion* & Categorical & Text & 421 & 27 & 6.4 & (4.3 to 9.2 ) \\
\hline Prostatic intraepithelial neoplasia* & Categorical & Text & 420 & 27 & 6.4 & (4.3 to 9.2$)$ \\
\hline Margins* & Binary & Text & 386 & 5 & 1.3 & (0.42 to 3.0$)$ \\
\hline Tumour volume & Continuous & Numeric & 310 & 4 & 1.3 & (0.35 to 3.3$)$ \\
\hline Prostate dimensions $†$ & Continuous & Numeric & 272 & 2 & 0.7 & (0.09 to 2.6$)$ \\
\hline Prostate weight & Continuous & Numeric & 410 & 5 & 1.2 & (0.40 to 2.8$)$ \\
\hline All fields & & & 5148 & 146 & 2.8 & (2.4 to 3.3$)$ \\
\hline
\end{tabular}

*Data required some interpretation on data entry-these were coded numerically.

†Each data point was a combination of three numbers. Error never occurred in more than one dimension.

$-(1-p)^{n}$, where $p$ is the overall error rate and $n$ is the number of fields. In this case, $1-(1-0.028)^{12}=31 \%$. Since the proportion of records where error occurred was $24 \%$, the errors appear not to occur independently across the fields.

Fields involving descriptive parameters appeared more error-prone than those with direct measurements or involving numerical figures, so we grouped the fields based on data format. Of the 2658 data points involving numbers (numeric and alphanumeric), 30 (1.1\%, 95\% CI 0.78 to 1.6 ) were erroneous, compared with 116 $(4.7 \%, 95 \%$ CI 3.9 to 5.6$)$ of the 2490 data points with text $(\mathrm{p}<0.0001)$. The five fields that required an element of interpretation in data entry also appeared more error-prone and again, when data were pooled, their difference in error rates compared with fields allowing for direct transcription was significantly greater $(5.2 \%$ vs $1.3 \%, \mathrm{p}<0.0001)$.

In the 971 cases used for the analysis of source data error, six cases were staged T2, but in fact were positive for extraprostatic extension (6 of $672,0.9 \%$ ). On pathologist review, these cases had indeed been understaged. One case of a T3 prostate cancer erroneously stated on the synoptic report that extraprostatic extension was not identified (table 3). This was the sole inconsistency between the original prose pathology report and its

Table 3 Analysis of errors in source pathology data was performed by matching stage with extraprostatic extension status (EPE). T2 disease should have been EPE negative, whereas T3 disease should have been EPE positive unless seminal vesicle involvement was documented

\begin{tabular}{lllll}
\hline $\begin{array}{l}\text { Pathological } \\
\text { stage }\end{array}$ & \multicolumn{5}{c}{ Matches } & \multicolumn{3}{c}{ Error } \\
\hline T2 & 672 & 6 & 0.9 & $(0.33$ to 1.9$)$ \\
T3 & 292 & 1 & 0.3 & $(0.01$ to 1.9$)$ \\
Total & 964 & 7 & 0.7 & $(0.30$ to 1.5$)$ \\
\hline
\end{tabular}

accompanying synoptic report (1 of 971, $0.1 \%)$. Although only two variables have been analysed, these figures suggest a very low rate of baseline error inherent in the pathology reports.

\section{DISCUSSION}

In a large contemporary radical prostatectomy dataset, we have examined pathology data in a subset of over 400 patients and found the overall error rate due to manual data entry to be $2.8 \%$ across all fields. Individual fields were found to vary in error rates between $0.5 \%$ and $6.4 \%$, and those involving descriptive text or requiring an element of interpretation appeared more vulnerable to error. Almost a quarter of patients had at least one data error when all pathology fields were considered, as might occur when multivariable statistical analysis is undertaken. We have also examined the source data electronically without human influence and established a baseline error rate of less than $1 \%$.

The strengths of our study include a combination of factors that enable a realistic representation of a small-to-moderate-sized oncology database used for research purposes. As the data were stored in a simple spreadsheet, not collected for clinical use and were sourced from primary clinical documents, this context of data entry represents a common scenario predating modern informatics solutions. We also examined a distinct set of data fields with varying formats important to clinical oncological research. Together, these increase the relevance of our findings to cancer datasets in general, and in particular to data which have been collected in times prior to the introduction of more sophisticated modes of data entry. We have also checked the integrity of one aspect of our source data, which is of importance in both clinical and academic settings. This helps to set a lower limit to the general error rate that can be achieved with interventions for data integrity imposed beyond initial pathology reporting. 
Our study was limited by its use of a single spreadsheet from a single series of patients. Although different institutions may use different data systems, the maintenance of clinical datasets on such spreadsheets is common in the clinical environment. Our source data were in the form of synoptic reports designed for the ease of data transcription, rather than traditional pathology reports in prose and this may have reduced the true error rate of such data. Other major limitations were in the study design, whereby we could not differentiate easily between different types of data entry error despite being able to infer this to some extent from the format of data. Owing to the nature of spreadsheets, we could not definitely account for row or column shifts in blocks of data as a source of error, although, on visual inspection of the errors, this did not seem to be the case. As we only examined pathology fields covered by electronic import, the findings were not representative of the entire dataset, which also includes operative and perioperative details, and thus the study was not designed to test the effect that these other factors may have had on error nor was it designed to detect errors in these important areas. A final limitation was that the size of error in fields containing continuous data was not measured as we only identified mismatches in the datasets, and this is required to assess more fully any impact of error in those fields.

Studies of error in clinical datasets are scarce, owing in part to the time and resources required to conduct these audits. Our overall error rate in manually entered data appears similar to that of previous studies. In one study occurring over 10 years, Zellner et $a l^{15}$ reported an estimated probability of error in two systems at about $2.4 \%$ and the estimated error frequency in a database alone was $2.7 \%$. In this case, less than $10 \%$ of the overall dataset was examined using random sampling. Arndt et $a l^{11}$ performed a detailed study of observer rating scores in a multicentre field setting, while Goldberg et $a l^{7}$ examined several clinical research databases, with errors ranging from $2.3 \%$ to $26.9 \%$ detected by the double-entry method in fields relating to timepoints of disease and tumour recurrence status. In general, these studies have involved more sophisticated data entry interfaces that allowed more detailed analysis of the underlying aetiology of data errors.

In contrast, our study has directly examined most fields in the subset of pathology variables, of particular importance in oncology research, and removed the effects of manual transfer of data in the generation of the comparison dataset. We also analysed the error in the source data, as they might exceed those of data entry and render attempts to decrease downstream error frequency less meaningful. In this case, the rate of $0.9 \%$ in mismatch between stage and extraprostatic extension was reassuringly lower than the overall manual entry dataset error frequency, and was also lower than the generally cited rate of $1.4 \%$ error for prostate pathology. ${ }^{16}$
Although an analysis of the impact of data errors on outcomes was an area our study was unable to address, as follow-up times were too short in our dataset for meaningful results, others have demonstrated the variable effects that erroneous data might have on outcomes such as rates of tumour recurrence and death rates. ${ }^{7} 8$ While it is likely that a low rate of data error will have little effect in univariable analysis, studies involving many fields and demonstrating a small effect size with borderline significance levels are intuitively liable to the effect of errors. In these cases, and particularly where accuracy across a large feature space is essential such as in translational genomics research, it is preferable that data errors are accounted for. Some investigators have developed corrective statistical tools to be used with a specified error rate in source databases in a particular circumstance, ${ }^{8}$ but such tools are unlikely to become widely applicable without more reporting of error rates within different types of datasets and analyses of outcome differences.

The greatest influence on error rates in our own clinical dataset was the transition to electronic data feeds from clinical sources and the application of software to retrospectively replace manually entered data. In doing so, we decreased the portion of patients with manually entered data from $58 \%(853 / 1471)$ to $9 \%(128 / 1471)$, although for various technical reasons many fields still remain manually inputted among the earlier patients. With advances in technology, it may be possible to extract data from even earlier pathology reports, since all reports are typewritten, and maintain a dataset with virtually no manually entered pathology data. Where such manually entered data are unavoidable or forms part of a larger dataset, due acknowledgement of the provenance of data from different parts of that dataset by performing separate analysis or by employing sensitivity analysis might be considered in research. In addition, the judicious selection of pathology fields based on liability to error might be used.

The recognition that direct use of clinically attained data leads to better accuracy is not new. The need to rekey data between clinical sources and database interfaces has long been acknowledged to be a significant source of human error, ${ }^{17}$ and the removal of this aspect of data entry would presumably increase the accuracy of clinical datasets overall. In recent times, the availability of data directly collected in the clinical setting for other healthcare activities including medical research has increased. One clinical group in a peripheral hospital centralised data collection for audit and research purposes via web-browser-based application software and extensively integrated the system in daily use. In just 12 months, their unit amassed over 3000 near complete patient records and reported enhanced accuracy due to the demonstration of the immediate clinical value of high-quality data capture to the users. ${ }^{18}$ Such integrated record systems have been shown to have additional clinical benefits, ${ }^{19}$ while data collected as near in time and 
space as possible to the point of care is known to improve the overall accuracy. ${ }^{20}$ Indeed, pathologists currently generate pathology reports prospectively as part of the clinical process, and our use of electronic data feeds from our service provider is an example of how clinical data can be directly captured for clinical or research use without the need for manual data entry.

With increasing drive for the widespread implementation of electronic health records ${ }^{21}$ comes the opportunity for more electronic data feeds into data repositories from health services such as those used in the present study, and this effectively diminishes the reliance on manual data entry. However, as our study demonstrated, even a clinical data source itself has a pervasive error rate, and there will remain a need for active error trapping. Retrospective replacement of manually entered data may also afford further opportunities to examine the errors in manually entered data. Such work might eventually prompt the development of tools to adequately account for errors in early datasets that no technology can correct.

\section{CONCLUSION}

We have evaluated a large radical prostatectomy dataset and while the overall rate of error was low, individual pathology fields were variably prone to error. We have demonstrated the feasibility of checking source clinical data for error, and the possibility of attaining high quality data using electronic data feeds for both prospective and retrospective parts of our data repository. We found that numerical data or data with fixed field entry provide better quality concordance between manual and electronic data-entry.

Acknowledgements Dr MKH Hong was supported by scholarships from the Royal Australasian College of Surgeons and from the National Health and Medical Research Council, Australia. We gratefully acknowledge Roberto Leiva for his technical assistance.

Contributors MKHH conceived and designed the study, interpreted the data and wrote the manuscript. HHIY participated in study design, data interpretation, critically reviewed the article, revised article and final approval. JSP participated in study design, acquired and analysed the data, critically reviewed the article and final approval. JSP acquired data, critically reviewed article and final approval. AJC acquired data, critically reviewed the article and final approval. DGM participated in conception and design of study, critically reviewed the article and final approval. CMH participated in study design, data interpretation, revised the article and final approval. NMC participated in study design and conception, acquisition of the data, interpretation of the data, revised the article and final approval.
Funding This research received no specific grant from any funding agency in the public, commercial or not-for-profit sectors.

\section{Competing interests None.}

Provenance and peer review Not commissioned; externally peer reviewed.

Data sharing statement No additional data are available.

\section{REFERENCES}

1. Hudson TJ, Anderson W, Artez A, et al. International network of cancer genome projects. Nature 2010;464:993-8.

2. Harel A, Dalah I, Pietrokovski S, et al. Omics data management and annotation. Methods Mol Biol 2011;719:71-96.

3. Needham DM, Sinopoli DJ, Dinglas VD, et al. Improving data quality control in quality improvement projects. Int $J$ Qual Health Care 2009;21:145-50.

4. Arts DG, De Keizer NF, Scheffer GJ. Defining and improving data quality in medical registries: a literature review, case study, and generic framework. J Am Med Inform Assoc 2002;9:600-11.

5. Beretta L, Aldrovandi V, Grandi E, et al. Improving the quality of data entry in a low-budget head injury database. Acta Neurochir (Wien) 2007;149:903-9.

6. Seddon DJ, Williams EM. Data quality in population-based cancer registration: an assessment of the Merseyside and Cheshire Cancer Registry. Br J Cancer 1997;76:667-74.

7. Goldberg SI, Niemierko A, Turchin A. Analysis of data errors in clinical research databases. AMIA Annu Symp Proc 2008:242-6.

8. Gallivan S, Pagel C. Modelling of errors in databases. Health Care Manag Sci 2008;11:35-40.

9. Goldberg SI, Niemierko A, Shubina M, et al. 'Summary Page': a novel tool that reduces omitted data in research databases. BMC Med Res Methodol 2010;10:91.

10. Warsi AA, White S, McCulloch P. Completeness of data entry in three cancer surgery databases. Eur J Surg Oncol 2002;28:850-6.

11. Arndt S, Tyrrell G, Woolson RF, et al. Effects of errors in a multicenter medical study: preventing misinterpreted data. J Psychiatr Res 1994;28:447-59.

12. Hayrinen K, Saranto K, Nykanen P. Definition, structure, content, use and impacts of electronic health records: a review of the research literature. Int J Med Inform 2008;77:291-304.

13. Antonarakis ES, Feng Z, Trock BJ, et al. The natural history of metastatic progression in men with prostate-specific antigen recurrence after radical prostatectomy: long-term follow-up. BJU Int 2012;109:32-9.

14. Neo EL, Beeke C, Price T, et al. South Australian clinical registry for metastatic colorectal cancer. ANZ J Surg 2011;81:352-7.

15. Zellner D, Schromm T, Frankewitsch T, et al. Structured data entry for reliable acquisition of pharmacokinetic data. Methods Inf Med 1996;35:261-4.

16. Frable WJ. Surgical pathology - second reviews, institutional reviews, audits, and correlations: what's out there? Error or diagnostic variation? Arch Pathol Lab Med 2006;130:620-5.

17. Gostel R. HyperCard to SPSS: improving data integrity. Comput Nurs 1993;11:25-8.

18. Tran $\mathrm{P}$, Morrison SG, Lade JA, et al. An integrated approach to surgical audit. ANZ J Surg 2011;81:313-14.

19. Featherstone I, Keen J. Do integrated record systems lead to integrated services? An observational study of a multi-professional system in a diabetes service. Int J Med Inform 2012;81:45-52.

20. Veen EJ, Janssen-Heijnen ML, Leenen LP, et al. The registration of complications in surgery: a learning curve. World $J$ Surg 2005;29:402-9.

21. Pearce C, Haikerwal MC. E-health in Australia: time to plunge into the 21st century. Med J Aust 2010;193:397-8. 